\title{
Fabrication of graded helical square tower-like Mn sculptured thin films and investigation of their electrical properties: comparison with perturbation theory
}

\author{
Mahsa Fakharpour ${ }^{1} \cdot$ Hadi Savaloni $^{2}$
}

Received: 16 January 2017/ Accepted: 6 February 2017/Published online: 20 February 2017

(c) The Author(s) 2017. This article is published with open access at Springerlink.com

\begin{abstract}
Mn sculptured thin films were fabricated in form of graded helical square tower-like terraced sculptured Mn thin films (GHSTTS) using oblique angle deposition together with rotation of substrate about its surface normal with fixed rotation angle $\left(90^{\circ}\right)$ and a shadowing block which was fixed at the centre of the substrate holder. The anisotropy of the samples was examined by resistivity measurements at two orthogonal angles. Direct relationship is obtained between resistivity and the anisotropy of the produced samples which showed that both of these parameters increase with decreasing distance from the edge of the shadowing block. Simulation work using the perturbation theory produced results consistent with the experimental observations.
\end{abstract}

Keywords Mn sculptured thin films · Anisotropy · Electrical property · Perturbation theory · Shadowing effect $\cdot$ Helical

\section{Introduction}

The size and shape of nano-particle as well as the environment (medium) are important parameters in determining the properties of the nano-particle. The different properties of the nano-particle or an assembly of nanoparticles in form of a thin film may be adjusted by

Hadi Savaloni

Savaloni@khayam.ut.ac.ir

1 Department of Physics, Science and Research Branch, Islamic Azad University, Tehran, Iran

2 Department of Physics, University of Tehran, North-Kargar Street, Tehran, Iran controlling the size and shape of the nano-particle(s). Hence, it is of utmost importance to have control on the fabrication process of such structures. Two of such techniques are proposed and implemented during the last few years namely oblique angle deposition (OAD) (vapor incident angle less than $85^{\circ}$ ) of thin films and glancing angle deposition (GLAD) (vapor incident angle greater than $85^{\circ}$ ) as physical vapor deposition methods have provided facilities for production of variety of nano-structures with structural anisotropy which can be controlled by predesign of the structure [1]. Once these techniques are combined with rotation of substrate in two different directions, two-dimensional and three-dimensional structures can be fabricated [2, 3] which is known as thin film nano-engineering. The main phenomenon in oblique angle is the shadowing effect. The self shadowing effect prohibits areas at the rear of the formed nuclei where the growth is extensively reduced $[4,5]$, and further deposition of atoms cause growth of these nuclei which ultimately tilted columnar structure with voids form on the substrate surface [6-8]. Sculptured thin films consist of columnar structures that their growth direction is being controlled/changed by movement of substrate. Complex structures such as chiral nano-flower shaped sculptured thin films are produced and their optical properties are investigated both experimentally [9] and theoretically [10-12].

Sculptured nano-structures fabricated by these methods have a vast area of applications such as optical filters [13], reflectors [14], polarizers [15], sensors [16, 17], bioscaffolds [18], and microchannels [19].

In this work graded helical square tower-like terrace shaped sculptured thin films (GHSTTS) were produced by oblique angle deposition together with the rotation of the substrate sequentially by $90^{\circ}$, while a shadowing block was fixed at the centre of the substrate holder having substrates 
mounted on it at different distances from the edge of the shadowing block. In addition, the length of the arms of the second and third pitches of the GHSTTS were reduced by a third and two thirds relative to the length of the arms of the first pitch; hence, terraced structures as well as slanted shapes in which the tilt angle changes with distance from the edge of the shadowing block were formed.

\section{Theory}

\section{Perturbation method for calculation of electrical resistance}

Hori and Yonezawa [20] in 1977 introduced a formalism in which a relationship is made between the electric field of a conductive material with voids (inhomogeneous region) and the electric field of that material with homogenized structure. The relationship between localized current density, conductivity and localized electric field in the introductory electromagnetism is given as follows:

$J_{i}(r)=\sigma(r) E_{i}(r)$,

If the region under examination consists of voids (as a perturbing medium), then one may introduce $\delta \sigma(r)$ as the local perturbation in the conductivity $\sigma(r)$;

$\sigma(r)=\sigma_{0}+\delta \sigma(r)$,

where $\sigma_{0}$ is conductivity of the material. Using the electrostatic potential $\Phi(r)$ and the continuity relationship, we have

$\Phi\left(r_{1}\right)=\Phi_{0}\left(r_{1}\right)+\int d r_{2} g\left(r_{12}\right) \frac{\partial}{\partial x_{2, i}}\left(\delta \sigma\left(r_{2}\right) \frac{\partial \Phi\left(r_{2}\right)}{\partial x_{2, i}}\right)$

where $r_{12}=r_{2}-r_{1}$ and the integral is taken over whole space $v$ or $s$. $\Phi_{0}(r)$ only depends on $\sigma_{0}$ and the boundary condition. The electric field is calculated as

$E_{i}\left(r_{1}\right)=E_{i}^{0}\left(r_{1}\right)+\int d r_{2} G_{i j}\left(r_{12}\right) \delta \sigma\left(r_{2}\right) E_{j}\left(r_{2}\right)$,

$E_{i}^{0}\left(r_{1}\right)$ is independent of $\delta \sigma\left(r_{1}\right)$ because it is the electric field of homogenized medium with $\sigma_{0}$ conductivity at $r_{1}$, $E_{i}\left(r_{1}\right)$ is the electric field of the $i$ th component at $r_{1}$ and $G_{i j}\left(r_{12}\right)$ is the Green function defined as

$$
\begin{aligned}
G_{i j}\left(r_{12}\right)= & -\frac{\partial^{2} g\left(r_{12}\right)}{\partial x_{1, i} \partial x_{2, j}} \\
& =\left\{\begin{array}{l}
-\frac{\delta_{i j} \delta\left(r_{12}\right)}{3 \sigma_{0}}+p \frac{1}{4 \pi \sigma_{0} r_{12}^{3}}\left(3 \frac{x_{12, i}}{r_{12}} \frac{x_{12, j}}{r_{12}}-\delta_{i j}\right) 3 D \\
-\frac{\delta_{i j} \delta\left(r_{12}\right)}{2 \sigma_{0}}+p \frac{1}{2 \pi \sigma_{0} r_{12}^{2}}\left(2 \frac{x_{12, i}}{r_{12}} \frac{x_{12, j}}{r_{12}}-\delta_{i j}\right) 2 D
\end{array}\right.
\end{aligned}
$$

In order to solve Eq. (4), we considered the thin film as a large rectangular cube consisting of many small cubes (see Fig. 2 in [21]). It may also be assumed that the electric field and conductivity in each of these small cubes is fixed (i.e. variation of these two quantities with respect to the small size of the cube is negligible). With these assumptions and some calculations, Eq. (4) becomes

$$
\begin{aligned}
E_{i}^{0}\left(r_{k}\right)= & E_{i}\left(r_{k}\right)\left(1+\frac{\delta \sigma\left(r_{k}\right)}{3 \sigma_{0}}\right) \\
& -\sum_{j=1}^{3} \sum_{l \neq k} v_{l} \frac{1}{4 \pi \sigma_{0} r_{k l}^{3}}\left(3 \frac{x_{k l, i}}{r_{k l}} \frac{x_{k l, j}}{r_{k l}}-\delta_{i j}\right) \delta \sigma\left(r_{l}\right) E_{j}\left(r_{l}\right),
\end{aligned}
$$

where $v_{l}$ is the volume of each small cube, $r_{k l}$ is the distance between $k$ th cube and $l$ th cube and $x_{k l, i}$ is the $i$ th component of vector connecting $r_{k}$ to $r_{l}$ whose value is $r_{k l}$. Since this value under no circumstance becomes zero, there is no need to use the principle value [21].

In addition, one should also consider some of these small cubes as voids (void fraction) and some of them as the film material (fraction of material inclusions). In order to implement this idea according to the film structure, one should consider some of the columns made of small cubes as the film material with $\sigma_{0}$ conductivity and the columns between them as voids with zero conductivity. In order to perform the calculations in this work, the dimensions of the cubes were considered as large enough that classical concepts can be applied [21]. At this stage, after considering the above operations, Eq. (6) transfers to $3 \mathrm{~N}$ equations with $3 \mathrm{~N}$ unknowns, where $\mathrm{N}$ is the number of small cubes and the components of local electric fields are the unknowns of the problem. After solving these $3 \mathrm{~N}$ equations the local current density can be obtained from multiplication of the electric field of each cube with its conductivity. The summation of local current density of all cubes will provide the total current density. Finally, multiplying the total current density with cross-section area of the thin film in a certain direction yields the resistance of the film. This resistance is the relative resistance of the film (i.e., the ratio of the resistance of the porous film to a homogeneous film from the same material). Hence in the perturbation theory the type of the material is not included and only the geometry of the structure is involved.

\section{Exprimental details}

The graded helical square tower-like (terraced) sculptured (GHSTTS) Mn thin films were deposited on glass (microscope slide) substrates by electron beam evaporation from a graphite crucible at room temperature. The purity of $\mathrm{Mn}$ was 99.99\%. An Edwards (Edwards E19 A3) coating plant with a base pressure of $2 \times 10^{-7}$ mbar was used. The 
deposition angle was fixed at $85^{\circ}$ and the substrate was rotated clock-wise for $90^{\circ}$ azimuthally for production of each arm of the GHSTTS. The substrate holder system is controlled by two stepping motors which can rotate the substrate holder by the two angles, $\alpha$ and $\varphi$, with $0.01 \%$ step accuracy and with controlled speed. The movement of the stepper motor for rotation of substrate about its surface normal $(\varphi)$ and its speed of revolution as well as facility for dividing each revolution to different sectors are controlled through interface to a computer in which the related software (in the LABVIEW format) is written and installed. All these are domestic made. The substrate holder was a stainless steel disc of $12 \mathrm{~cm}$ diameter. At the centre of this disc a cylindrical block $(2 \mathrm{~cm}$ in diameter and $2 \mathrm{~cm}$ in height) was fixed as a shadowing block. The substrates were fixed at 1, 3 and $5 \mathrm{~cm}$ distance from this shadowing block along four mutually normal radii of the substrate holder disc. Hence, in each run four sets of three samples were produced for use in different analyses and reproducibility check of the samples.

Three different GHSTTS Mn thin films consisting of different number of arms, namely eight, nine and ten arms were produced. Each arm of the first pitch was deposited for $110 \mathrm{~nm}$ while each arm of the second and third pitches was 72 and $36 \mathrm{~nm}$, respectively.

The deposition rate was fixed at $1.0 \AA^{-1}$. The deposition rate was measured by a quartz crystal deposition rate controller (Sigma Instruments, SQM-160, USA) positioned close to the substrate holder and at almost the same azimuthal angle as that of the substrate. This was calibrated after obtaining the film thickness using a field emission electron microscope (FESEM) and dividing by the deposition time. The distance between the evaporation source and the substrate was $30 \mathrm{~cm}$. In this arrangement, the vapor source ( $6 \mathrm{~mm}$ in diameter) behaves like a point source with a cosine distribution, and because of the $30 \mathrm{~cm}$ distance between the vapor source and the substrate it is expected that the vapor has straight trajectories (i.e., no appreciable scattering due to the large mean free path $(\sim 103-104 \mathrm{~cm}$ [22]) occurs).

Prior to deposition, all glass substrates were ultrasonically cleaned in heated acetone and then ethanol. The surface roughness of the substrates was measured by a Talysurf profilometer and a AFM and the rms (route mean square) substrate roughness $(\mathrm{Rq})$ obtained using these methods were 0.3 and $0.9 \mathrm{~nm}$, respectively.

The deposition process was repeated a few times and the reproducibility of the results was confirmed. The film thicknesses and column shapes and sizes were measured by field emission electron microscope (FESEM) (Hitachi S-4100 SEM, Japan). The FESEM samples were coated with a very thin layer of gold to prevent the charging effect.

The surface physical morphology and surface roughness of the samples were obtained by means of AFM (NT-MDT, SOLVER, Nova Tech) analysis with a Si tip of $10 \mathrm{~nm}$ diameter and in non-contact mode. The electrical measurements of these thin films were obtained by four point probe.
Fig. 1 FESEM images of graded helical square tower-like (terraced) Mn sculptured thin films produced at different distances from the edge of the shadowing block with different number of arms; column

(I) $1 \mathrm{~cm}$ distance; column (II)

$3 \mathrm{~cm}$ distance; column (III)

$5 \mathrm{~cm}$ distance. Row

(a) 10 arms; Row (b) 9 arms; Row (c) 8 arms

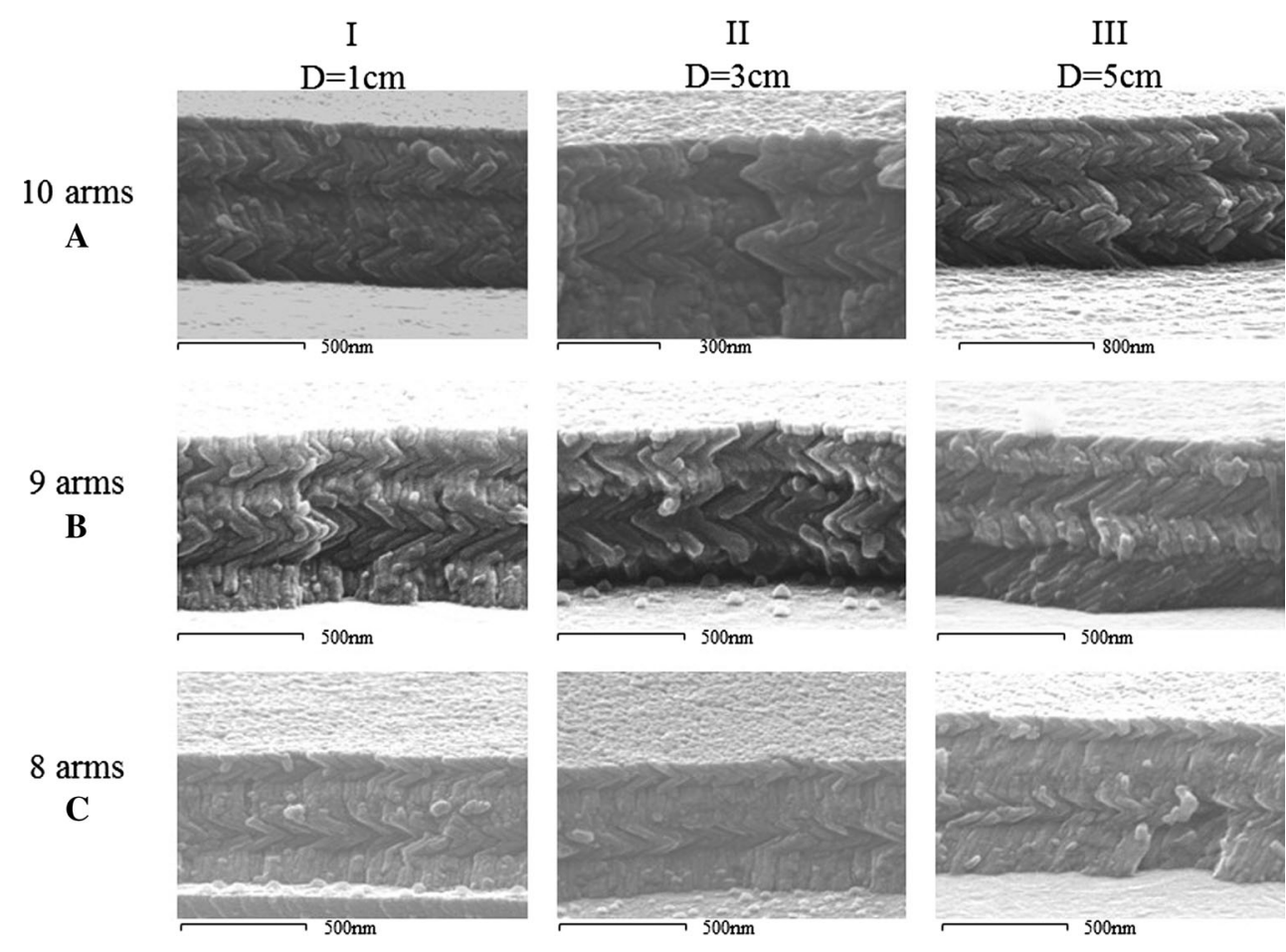


Table 1 Details of graded helical sculptured Mn thin film samples and their different parameters

\begin{tabular}{llllrrrr}
\hline Sample & $\begin{array}{l}\text { Distance from } \\
\text { the shadowing } \\
\text { block }(\mathrm{cm})\end{array}$ & Film thickness $(\mathrm{nm})$ & $\begin{array}{l}\text { Helical column } \\
\text { tilt angle }(\alpha \mathrm{\alpha t})\left({ }^{\circ}\right)\end{array}$ & $\mathrm{D}_{\mathrm{AFM}}(\mathrm{nm})$ & $\mathrm{R}_{\text {ave }}(\mathrm{nm})$ & $\mathrm{R}_{\mathrm{rms}}(\mathrm{nm})$ & $\mathrm{VF}_{\text {surf }}(\%)$ \\
\hline A1 & 1 & 465.5 & 14 & 122.0 & 1.75 & 2.24 \\
A3 & 3 & 504.1 & 8 & 112.0 & 1.33 & 1.68 \\
A5 & 5 & 550.8 & 4 & 96.0 & 1.20 & 1.50 & 13.32 \\
B1 & 1 & 436.7 & 12 & 120.7 & 2.33 & 2.87 \\
B3 & 3 & 450.0 & 8 & 109.6 & 2.20 & 2.86 \\
B5 & 5 & 544.8 & 4 & 81.0 & 1.96 & 2.58 \\
C1 & 1 & 417.4 & 12 & 132.3 & 2.60 & 19.04 \\
C3 & 3 & 441.0 & 5 & 128.9 & 2.57 & 3.42 \\
C5 & 5 & 525.0 & 3 & 91.9 & 2.03 & 3.28 \\
\hline
\end{tabular}

$D_{A F M}$ grain size obtained from AFM analysis. $R_{\text {ave }}$ and $R_{r m s}$ average and root mean square surface roughnesses, respectively, $V F_{\text {surf }}$ surface void fraction

\section{Results and discussion}

\section{Structure and surface morphology of GHSTTS thin films}

In Fig. 1 (columns I to III) the FESEM images of the cross sections of the GHSTTS Mn thin films with eight, nine and ten arms produced at three different distances of 1,3 and $5 \mathrm{~cm}$ from the edge of the shadowing block are shown. The helical structure of the grown nano-columns and the dense bottom layer of the films [23, 24] can clearly be distinguished in these images, while the grown arms of the square pitches and their angle as well as the growth angle of the columns (tilt angle) are also seen. In addition, one can also see the reduction of the length of the arms from bottom pitch to upper pitches consistent with the deposition procedure (110, 72 and $36 \mathrm{~nm}$ lengths of arms for the bottom, middle and top pitches, respectively). The tilt angle of the helical columns is measured from the FESEM images and is given in Table 1 for GHSTTSs with eight, nine and ten arms as mentioned above. In order to clearly identify the produced samples with three different number of arms and that each set contains of three different samples which were positioned at three different distances from the edge of the shadowing block (i.e., 1, 3 and $5 \mathrm{~cm}$ ) we have assigned the following names/symbols to them; the set with $10 \mathrm{arms}$ are assigned as " $A$ " group followed by distance from the shadowing block in centimeters: A1, A3 and A5. Similarly for the set with 9 arms we assigned them as B1, B3 and B5 and for the group with 8 arms we named them as C1, C3 and C5. Results of growth/tilt angle of columns and the thickness of the films measured from the FESEM images are given in Table 1 and these are consistent with the calculated results.
Figure 2i-iii shows the 2D and 3D AFM images (columns I and II) as well as surface void/inclusion fractions (column III), while in Table 1 the results of grain size distribution, percentage of surface void fraction (measured using JMicroVision software) and also average and root mean square surface roughness for all samples produced in this work obtained from the AFM results are given.

\section{Electrical resistivity}

The electrical resistivity of the Mn GHSTTS films produced in this work was measured using a four point probe instrument to obtain their I-V curves. Measurements were carried out at four different directions of $x$ (vapour incident direction) and y axes of the sample as well as two diagonal directions. These measurements provide us with the required data to evaluate the degree of anisotropy in the structure of the produced samples due to the structural differences (tilt angle, number of arms) and the bundling effect in these obliquely deposited thin films [25]. Resistivity of the films was calculated using [26]:

$\rho=\frac{2 \pi s V}{I}$,

The uncertainty of these results was obtained as [27]:

$\Delta \rho=\frac{\pi t}{\ln 2}\left(\frac{v}{I}\right) \sqrt{\left(\frac{\Delta v}{v}\right)^{2}+\left(\frac{\Delta I}{I}\right)^{2}}$.

As mentioned earlier due to anisotropic structure of these films their anisotropy was achieved using

Anisotropy $=\frac{\rho_{x}-\rho_{y}}{\rho_{x}+\rho_{y}}$.

In Fig. 3 results obtained for the I-V curves of the Mn GHSTTS thin films with 8, 9 and 10 arms deposited at 


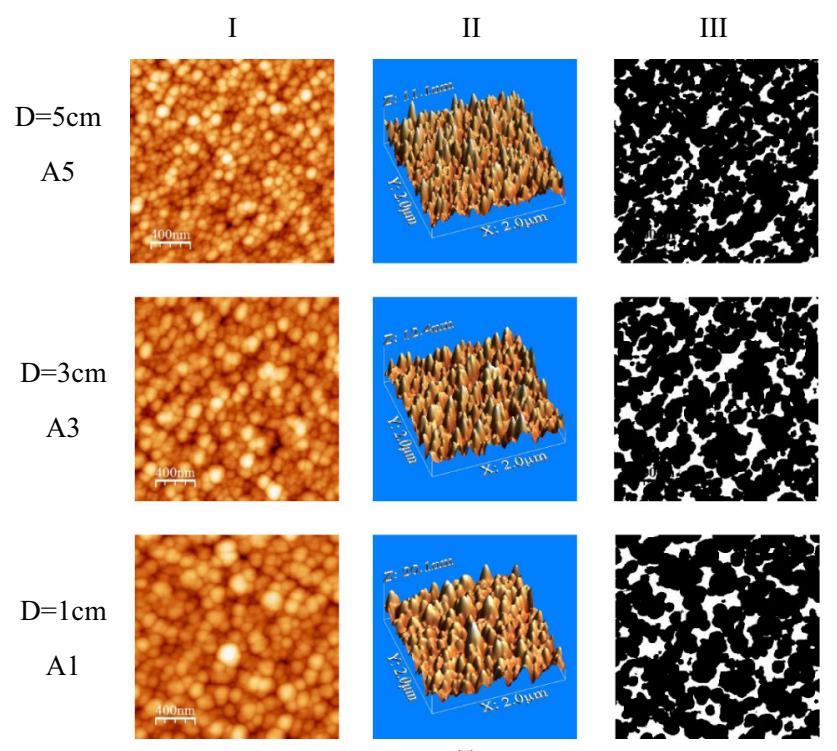

(I)
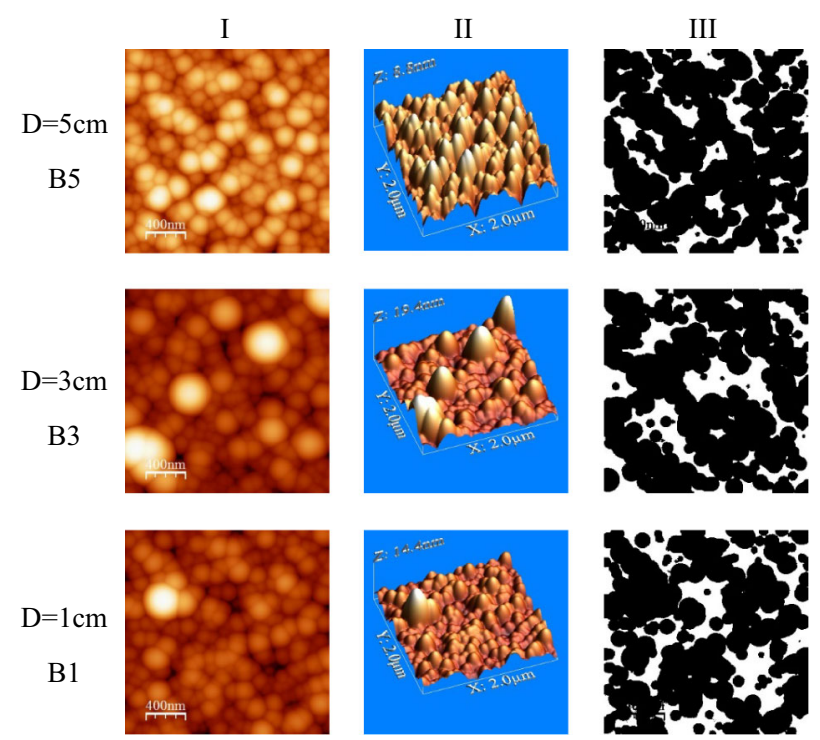

(II)

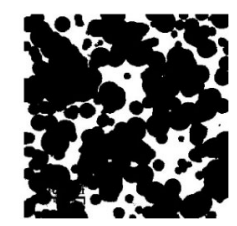

II
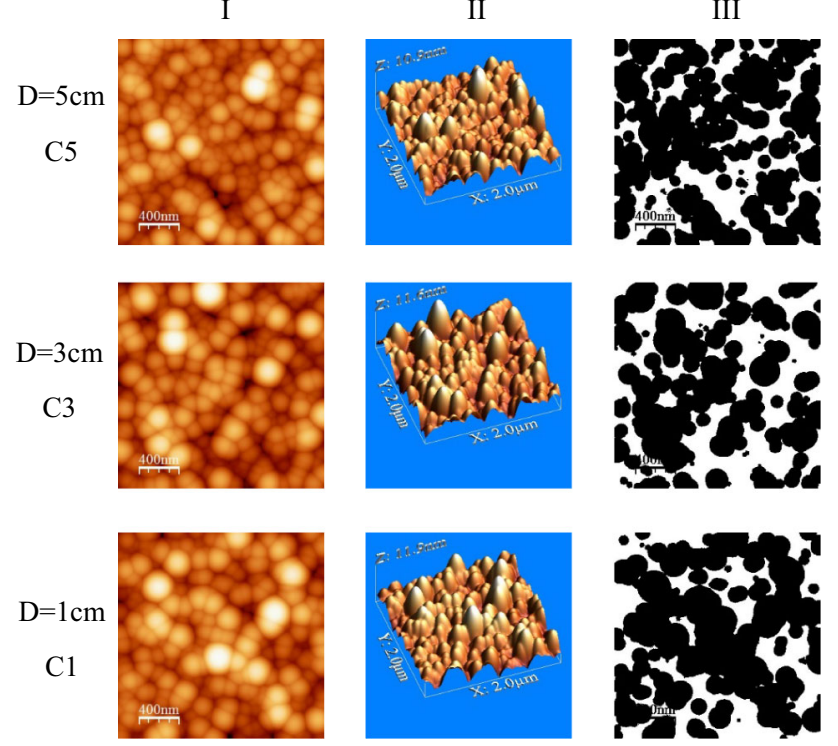

(III)

Fig. 2 (i) AFM images of graded helical square tower-like (terraced) Mn sculptured thin films produced at different distances from the edge of the shadowing block with 10 arms; column (I) 2D images; column (II) 3D images; column (III) void fraction. Row (a) $5 \mathrm{~cm}$ distance; Row (b) $3 \mathrm{~cm}$ distance; Row (c) $1 \mathrm{~cm}$ distance. (ii) AFM images of graded helical square tower-like (terraced) Mn sculptured thin films produced at different distances from the edge of the shadowing block with 9 arms; column (I) 2D images; column (II) 3D

different distances from the edge of the shadowing block in both $\mathrm{x}$ and $\mathrm{y}$ directions and two diagonal directions obtained from Eq. 7 are given. The influential parameters on the variation of resistivity can be surface porosity, surface roughness, film thickness or the length of the arms in the structures produced in this work, geometry of the structure and grain sizes. Now we may examine our results

images; column (III) void fraction. Row (a) $5 \mathrm{~cm}$ distance; Row (b) $3 \mathrm{~cm}$ distance; Row (c) $1 \mathrm{~cm}$ distance. (iii) AFM images of graded helical square tower-like(terraced) Mn sculptured thin films produced at different distances from the edge of the shadowing block with 8 arms; column (I) 2D images; column (II) 3D images; column(III) void fraction. Row (a) $5 \mathrm{~cm}$ distance; Row (b) $3 \mathrm{~cm}$ distance; Row (c) $1 \mathrm{~cm}$ distance

of the resistivity in Fig. 3. Considering the aforementioned parameters in relationship to the structure of different samples produced in this work, one can perform two types of comparison (analyses): (1) comparison of the resistivity of each structure (i.e., GHSTTS with 10, or 9 or 8 arms), with increasing distance from the edge of the shadowing block; (2) comparison of the three different GHSTTS with 


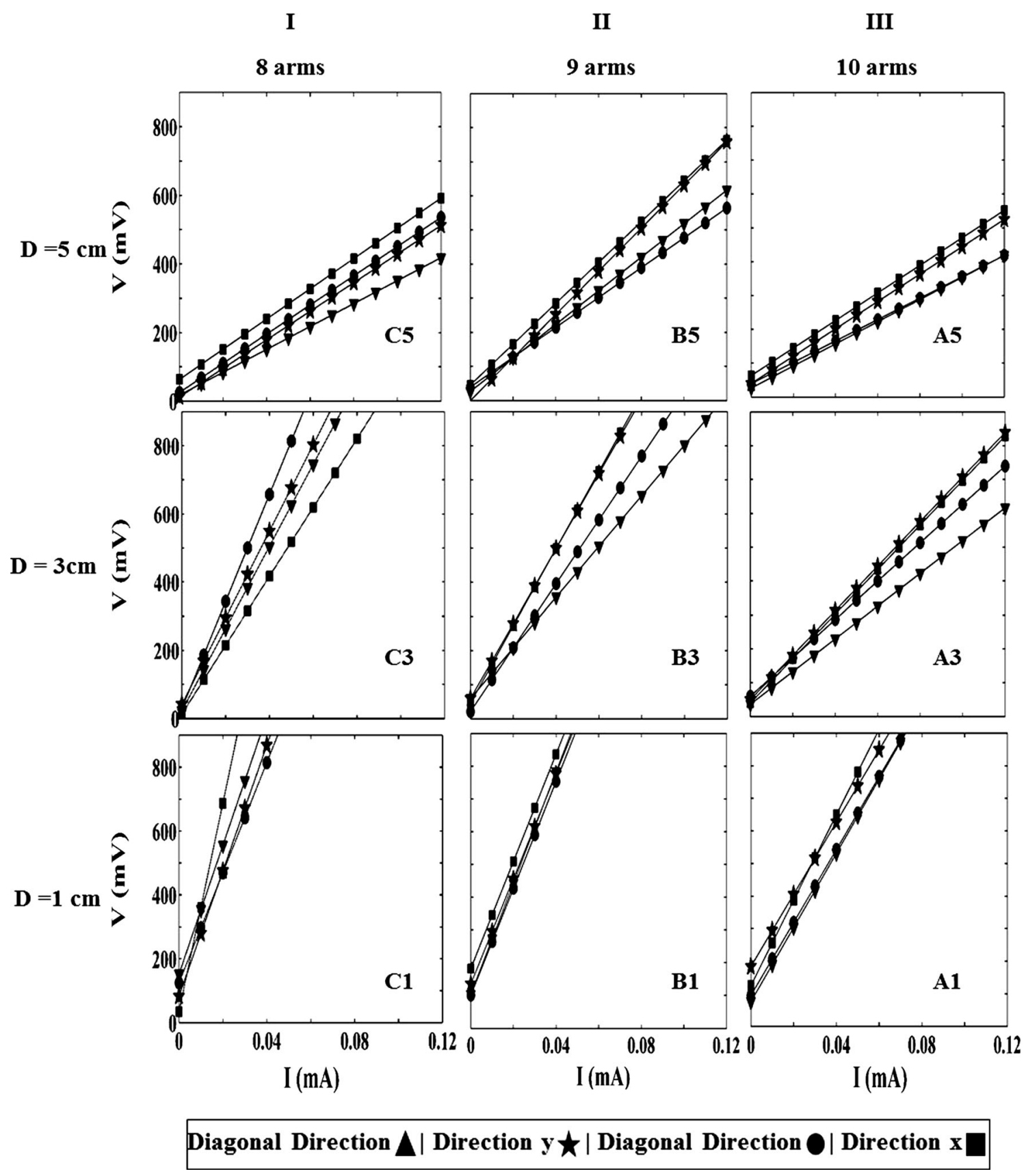

Fig. 3 I-V curves for GHSTTS structures with 8, 9 and 10 arms

the same distance from the edge of the shadowing block, but with different number of arms.

Comparison of samples of each structure with increasing distance from the edge of the shadowing block

The following observations may be made:

(a) The slop of the I-V curves is different for different measurement directions; hence the film structure is inhomogeneous and anisotropic which will be presented in the following paragraphs.

(b) The slop of I-V curves for the samples closer to the edge of the shadowing block is higher than those at further distance which reduces with increasing this distance. This shows that samples grown at close distance to the edge of the shadowing block have higher anisotropy/resistance. This in fact can be due to several factors as follows: the higher tilt angle of columns in this type of samples (i.e., A1, B1 and C1) relative to samples deposited at further distance 


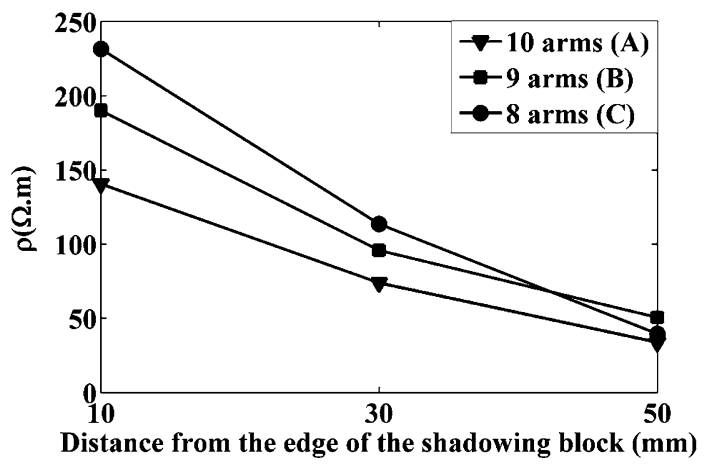

Fig. 4 Resistivity of GHSTTS structures with different number of arms versus distance from the edge of the shadowing block

(Table 1); higher void fraction of this type of samples obtained from the analysis of the AFM results (Table 1); higher surface roughness of these samples (Table 1), and the lower film thickness (Table 1).

\section{Comparison of the resistivity of GHSTTS with different number of arms at the same distance from the edge of the shadowing block}

In Fig. 4 the resistivity of $\mathrm{A}, \mathrm{B}$ and $\mathrm{C}$ samples positioned at different distances from the edge of the shadowing block are compared. It can be observed that the resistivity of these samples varies as follows: $\rho A 5 \prec \rho C 5 \prec \rho B 5, \rho A 3 \prec$ $\rho B 3 \prec \rho C 3, \rho A 1 \prec \rho B 1 \prec \rho C 1$. The order/trend of variation of the resistivity for the samples positioned at $5 \mathrm{~cm}$ distance from the edge of the shadowing block differs from the other two sets of samples though this difference is very small and may be ignored as it could be either due to the uncertainty in the data or due to the lower tilt angle of the helical columns for the C5 sample (see Table 1).

The general trend of variation seen in Fig. 4 can be explained on the bases (with reference to the data presented in Table 1) of the thickness of the films (the thicker films should have lower resistivity), surface roughness of the samples (surface roughness is decreased with increasing the number of arms, hence less electron scattering from surface roughness may occur which in turn reduces the resistivity of the sample) and void fraction of the samples (void fraction is increased for the samples closer to the edge of the shadowing block; hence it can be assumed that large potential barriers are produced between the grains which obstructs the movement of electrons along the length of the sample (or the surface).

\section{Electrical anisotropy of GHSTTS thin films}

In Fig. 5 results obtained for the resistivity of the GHSTTS nano-structures with 8,9 and 10 arms in both $\mathrm{x}$ and $\mathrm{y}$
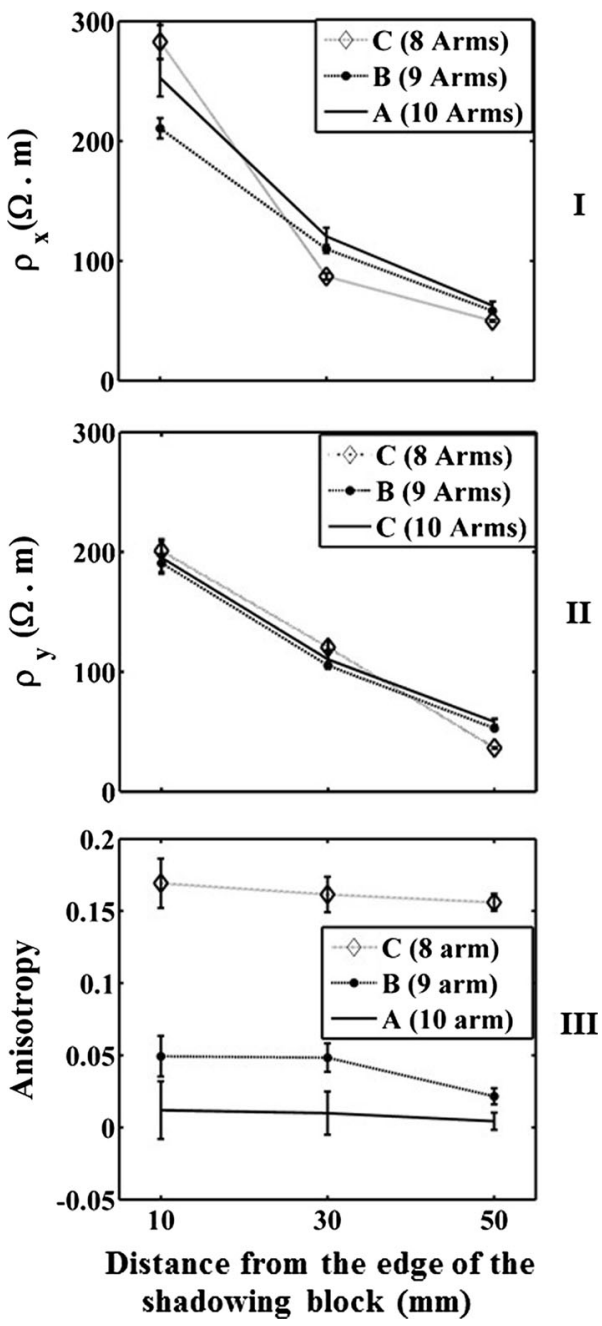

Fig. 5 Resistivity of GHSTTS structures with 8, 9 and 10 arms measured at two $x$ and $y$ directions and the obtained anisotropy

directions and the anisotropy of the samples obtained from Eq. (9) are given. It can be seen that there is a decreasing behaviour for all of these plots with increasing the distance from the edge of the shadowing block. Hence, the electrical measurements give us a proof and degree of anisotropy in the structure of the GHSTTS films produced in this work.

\section{Estimating the resistance of GHSTTS thin films}

As mentioned before, in order to calculate the relative resistivity of the samples produced in this work, the thin film was assumed in shape of a rectangle with $a \times b \times h$ dimensions. Then this rectangle was divided into many small cubes with a side length of $d$. It should be mentioned that dimensions of the rectangle are multiplication of the small cubes side length $d$. Hence, this procedure provides the required condition for working with dimensionless parameters/lengths. Then the geometry of 


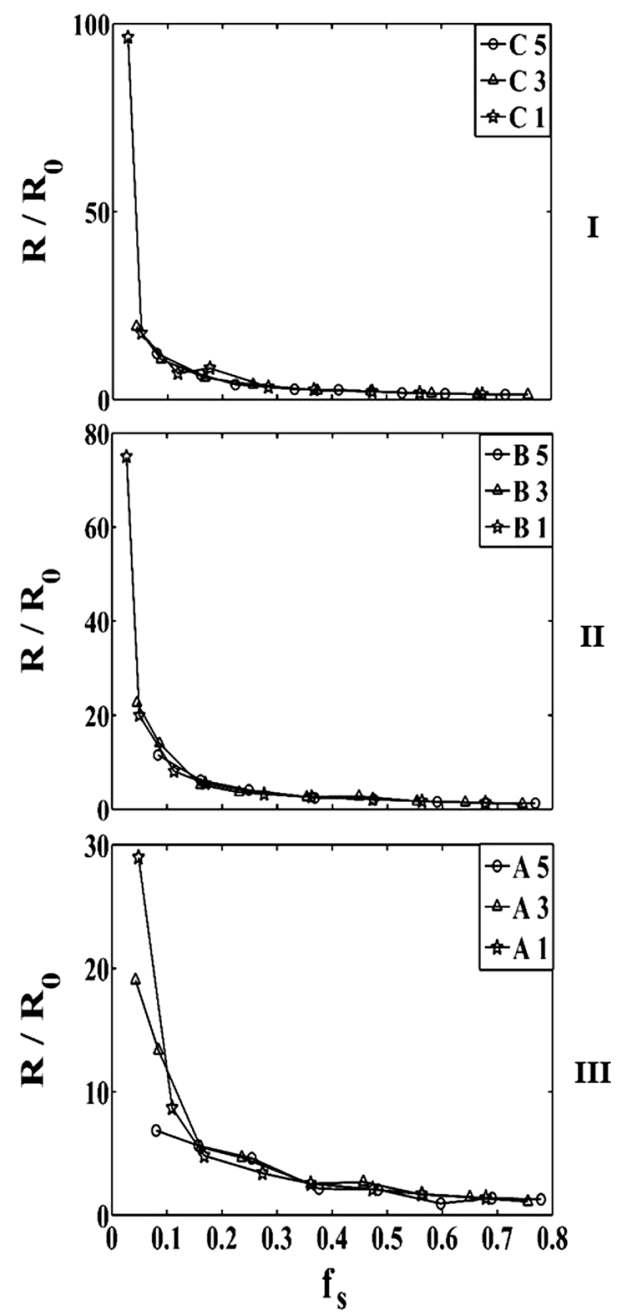

Fig. 6 Relative resistivity of GHSTTS structures with 8, 9 and 10 arms as a function of void fraction for different distances from the edge of the shadowing block

the structure is considered (i.e., some cubes were chosen as the material inclusion while they make the helical structure of GHSTTS with $\sigma_{0}$ conductivity. The remaining cubes produce the voids between the material inclusions with zero conductivity). The GHSTTS structure was made with different arm lengths so that in the first pitch the length of arms was $2 l$ and in the second pitch it was reduced to two third (i.e., 2l/3) and in the third pitch was reduced to onethird of the length of arm in the first pitch (i.e., l/3). Hence in the perturbation simulation work, the same procedure was assumed as in the first pitch the length of arm was taken as $6 d$, and in the second pitch it was $4 d$ and in the last (top) pitch it was $2 d$. Hence a cube is chosen with $6 d \times 6 d \times h$ dimensions. The structure of GHSTTS with 8,9 and 10 arms is the same and the only difference between them is the number of arms; hence, the thickness/ height of the cube in these samples is different which is included in the $h$ parameter. The thickness of the film was obtained by considering the tilt angle of the helical columns and the deposition angle and the length of the arms. Therefore, it is expected that the relative resistivity obtained from the perturbation theory/program does not show a large variation as the result of this program does not depend on the thin film dimensions and only depends on the geometry of the structure and the void fraction. Hence, as discussed in the preceding section in this calculation, for each value of material inclusion, $6 d \times 6 d \times h$ cubes are involved and a system of $3 \times 6 d \times 6 d \times h$ equations with the same number of unknowns is solved. We emphasise again that resistance calculations using this procedure give us the resistance of a columnar structure relative to a homogeneous structure as a function of material inclusion and the growth/rise angle of columns, while effects due to size/dimensions must be considered separately.

Results of relative resistivity as a function of void fraction obtained from calculations are given in Fig. 6. It can be seen that in the GHSTTS structures resistance increases with decreasing material inclusion. This is consistent with Siabi-Garjan et al.s' results for columnar structures [28]. In addition, from comparison of sub-figures in Fig. 6 it can be deduced that the relative resistivity decreases with increasing the number of arms in the GHSTTS structure. This is in agreement with the experimental measurements discussed in the preceding section (Fig. 4). Hence, the simulation work of the perturbation method clearly shows the influence of the geometrical shape of the structure on the electrical resistivity.

\section{Conclusion}

Graded helical square tower-like (terraced) sculptured (GHSTTS) Mn thin films were produced by deposition at oblique angle, rotation of substrate about its surface normal with fixed rotation angle $\left(90^{\circ}\right)$ and use of a shadowing block at the centre of the substrate holder. The produced films are anisotropic due to use of shadowing block which also leads to formation of graded structures. The variation of this anisotropy influences the electrical properties of the GHSTTS. The anisotropy of the samples was obtained by measuring the electrical resistivity of the samples at two orthogonal $x$ and $y$ directions. Results showed that the electrical resistivity and the anisotropy of the structure increase with decreasing distance from the edge of the shadowing block. This observation was confirmed by use of perturbation theory.

Acknowledgements This work was carried out with the support of the University of Tehran and the Islamic Azad University. HS is grateful to the Centre of Excellence for Physics of Structure and Microscopic Properties of Matter, Department of Physics, University 
of Tehran, for partial support of this work. Partial support of this work by Iran National Science Foundation (INSF) is greatly appreciated.

Open Access This article is distributed under the terms of the Creative Commons Attribution 4.0 International License (http://crea tivecommons.org/licenses/by/4.0/), which permits unrestricted use, distribution, and reproduction in any medium, provided you give appropriate credit to the original author(s) and the source, provide a link to the Creative Commons license, and indicate if changes were made.

\section{References}

1. Hawkeye, M., Brett, M.J.: Glancing angle deposition: fabrication, properties, and applications of micro-and nanostructured thin films. J. Vac. Sci. Technol. A 25(5), 1317-1335 (2007)

2. Jensen, M.O., Brett, M.J.: Periodically structured glancing angle deposition thin films. IEEE Trans. Nanotechnol. 4(2), 269-277 (2005)

3. Li, H.F., Kar, A.K., Parker, T., Wang, G.C., Lu, T.M.: The morphology and texture of $\mathrm{Cu}$ nanorod films grown by controlling the directional flux in physical vapor deposition. Nanotechnology 19(33), 335708 (2008)

4. Wang, F., Lakhtakia, A.: Response of slanted chiral sculptured thin films to dipolar sources. Opt. Commun. 235(1), 133-151 (2004)

5. Wang, F., Lakhtakia, A.: Lateral shifts of optical beams on reflection by slanted chiral sculptured thin films. Opt. Commun. 235(1), 107-132 (2004)

6. Nieuwenhuizen, J.M., Haanstra, H.B.: Microfractography of thin films. Philips Tech. Rev. 27(3), 87-91 (1966)

7. Messier, R., Yehoda, J.E.: Geometry of thin-film morphology. J. Appl. Phys. 58(10), 3739-3746 (1985)

8. Motohiro, T., Taga, Y.: Thin film retardation plate by oblique deposition. Appl. Opt. 28(13), 2466-2482 (1989)

9. Savaloni, H., Haydari-Nasab, F., Malmir, M.: Nano-structural characteristics and optical properties of silver chiral nano-flower sculptured thin films. Appl. Surf. Sci. 257(21), 9044-9055 (2011)

10. Siabi-Garjan, A., Savaloni, H.: Extinction spectra and electric field enhancement of silver chiral nano-flower shaped nanoparticle; comparison of discrete dipole approximation results with experimental results. Eur. Phys. J. B 86(6), 257 (2013)

11. Abdi, F., Siabi-Garjan, A., Savaloni, H.: Investigation on the dependence of optical spectra of silver chiral nanostructures on shape, dimensions and incident light by discrete dipole approximation. J. Theor. Appl. Phys. 6(1), 1-7 (2012)

12. Abdi, F., Siabi-Gerjan, A., Savaloni, H.: On the discrete dipole approximation investigation of the extinction spectra of $\mathrm{Ag} / \mathrm{glass}$ nano-flower thin film with threefold symmetry. J. Theor. Appl. Phys. 6(1), 4 (2012)

13. Lakhtakia, A., Messier, R.: Sculptured Thin Films: Nanoengineered Morphologyand Optics, Messier, p. 233. SPIE, Bellingham (2005)

14. Robbie, K., Brett, M.J.: Chiral sculptured thin films. Nature 38, 4616 (1996)

15. Hodgkinson, I.J., Wu, Q., Arnold, M., McCall, W., Lakhtakia, A.: Chiral mirror and optical resonator designs for circularly polarized light: suppression of cross-polarized reflectances and transmittances. Opt. Commun. 210(3), 201-211 (2002)

16. Steele, J.J., Van popta, A.C., Hawkeye, M.M., Sit, J.C., Brett, M.J.: Nanostructured gradient index optical filter for high-speed humidity sensing. Sens. Actuators B120(1), 213-219 (2006)

17. Kesapragada, S.V., Victor, O.N.P., Gall, D.: Nanospring pressure sensors grown by glancing angle deposition. Nano Lett. 6(4), 854-857 (2006)

18. Harris, K.D., Brett, M.J., Smy, T.J., Backhouse, C.: Microchannel surface area enhancement using porous thin films. J. Electrochem. Soc. 147(5), 2002-2006 (2000)

19. Lakhtakia, A., Demirel, M., Horn, M., Xu, J.: Six emerging directions in sculptured-thin-film research. Adv. Solid State Phys. 46, 295-307 (2007)

20. Hori, M., Yonezawa, F.: Theoretical approaches to inhomogeneous transport in disordered media. J. Phys. C 10(2), 229 (1977)

21. Siabi-Garjan, A., Savaloni, H., Beik-Mohammadi, J., GrayeliKorpi, A.-R.: On the characterization of sculptured columnar Mn thin films; correlation between nanostructure and the optical and electrical properties using experimental and simulation results. Philos. Mag. 93(26), 3527-3546 (2013)

22. Eckertova, L.: Physics of Thin Films, 2nd edn. Plenum Press, Berlin (1986)

23. Savaloni, H., Babaei, F., Song, S., Placido, F.: Characteristics of sculptured $\mathrm{Cu}$ thin films and their optical properties as a function of deposition rate. Appl. Surf. Sci. 255, 8041-8047 (2009)

24. Alouach, H., Mankey, G.J.: Texture orientation of glancing angle deposited copper nanowire arrays. J. Vac. Sci. Technol. A 22(4), 1379-1382 (2004)

25. Abelmann, L., Lodder, C.: Oblique evaporation and surface diffusion. Thin Solid Films 305(1-2), 1-21 (1997)

26. Taylor, J.R.: An Introduction to Error Analysis, 2nd edn. University Science Books, Sausalito (1997)

27. Savaloni, H., Babaei, F., Song, S., Placido, F.: Influence of substrate rotation speed on the nanostructure of sculptured $\mathrm{Cu}$ thin films. Vacuum 85(7), 776-781 (2011)

28. Siabi-Garjan, A., Savaloni, H.: Extinction spectra and electric near-field distribution of $\mathrm{Mn}$ nano-rod based sculptured thin films: experimental and discrete dipole approximation results. Plasmonics 10(4), 861-872 (2015) 